Pbilosophia Philosophia Scientiæ

Scientia

Travaux d'histoire et de philosophie des sciences

9-2 | 2005

Aperçus philosophiques en logique et en

mathématiques

\title{
Falsity Conditions for IF-Sentences
}

\section{Francien Dechesne}

\section{(2) OpenEdition \\ 1 Journals}

Electronic version

URL: http://journals.openedition.org/philosophiascientiae/549

DOI: $10.4000 /$ philosophiascientiae.549

ISSN: $1775-4283$

Publisher

Éditions Kimé

\section{Printed version}

Date of publication: 1 November 2005

Number of pages: $305-322$

ISBN: 2-84174-379-9

ISSN: $1281-2463$

Electronic reference

Francien Dechesne, "Falsity Conditions for IF-Sentences", Philosophia Scientiæ [Online], 9-2 | 2005,

Online since 15 June 2011, connection on 15 January 2021. URL: http://journals.openedition.org/

philosophiascientiae/549; DOI: https://doi.org/10.4000/philosophiascientiae.549 


\section{Falsity Conditions for IF-Sentences}

Francien Dechesne*
Department of Philosophy, Tilburg University, and
Department of Computer Science, Technische Universiteit Eindhoven,
(The Netherlands)

Abstract: We give a procedure to obtain falsity conditions for IF-sentences, using Skolemization. The expressive power of an IF-sentence can then be strongly captured by a pair of $\Sigma_{1}^{1}$-sentences. A result from [Burgess 2003] shows that, conversely, any pair of incompatible $\Sigma_{1}^{1}$-sentences corresponds with an IF-sentence.

In the second part, we reflect on the influence of the order of the steps (inside-out versus outside-in) in the Skolemization procedures for IF-logic. We also reflect on the nature of game theoretical negation.

*. The research project of the author is supported by the Samenwerkingsorgaan Brabantse Universiteiten (SOBU).

Philosophia Scientice, 9 Cahier 2, 2005, 301-318. 


\section{Introduction}

In his book The Principles of Mathematics Revisited ([Hintikka 1996]), Jaakko Hintikka proposes Independence Friendly Logic (IF-logic) with Game Theoretical Semantics as the logic that could open new directions in the foundations of mathematics.

IF-logic uses the syntax of classical first order logic, with an additional slash operator. This operator removes the slashed quantification or connective from the (syntactic) scope of the quantifications that appear under the slash. Semantically, the first becomes independent of the latter. For example, in the IF-formula $\forall x \exists y_{/ x}[x=y]$, the value of $y$ may not depend on the value of $x$.

Game Theoretical Semantics (GTS) associates with every IF-sentence $\varphi$ and suitable model $M$ a semantical game $G_{M}(\varphi)$, played by two players: Eloïse, who starts the game in the role of Verifier, and Abélard, who starts in the role of Falsifier.

Moves in the game are choices for domain elements as assignments for the variables bound by the quantifiers $\exists$ (move for player in the role of Verifier) and $\forall$ (move for the player in the role of Falsifier) and choices for 'left' or 'right', associated with the connectives $\vee$ and $\wedge$ (move for Verifier and Falsifier respectively). The negation sign $\sim$ makes the two players change roles.

The structure of the game is fixed by the syntax of the formula without the slashes: the slash operator induces restrictions on the level of the strategies. A strategic choice associated with a slashed quantifier or connective, cannot be based on the moves associated with the variables under the slash. We could say that the choice is made by the player, without knowing the values of the variables under the slash.

Truth and falsity of a sentence $\varphi$ in a model $M$ are defined as follows:

$$
\begin{aligned}
M \| \models_{\mathrm{t}} \varphi & :=\varphi \text { is true in } M \\
& :=\text { there exists a winning strategy for Elö̈se in } G_{M}(\varphi) \\
M \| \models_{\mathrm{f}} \varphi & :=\varphi \text { is false in } M \\
& :=\text { there exists a winning strategy for Abélard in } G_{M}(\varphi)
\end{aligned}
$$

It is a well known fact that finite two-player win-loss games of perfect information are determined, in the sense that one of the players has a winning strategy. By this fact, GTS makes first order formulas always either true or false.

For IF-formulas, this is no longer the case: the interpretation of the slash operator in GTS turns the semantical games into games of 
imperfect information. In that type of games, it can be the case that none of the two players has a winning strategy in a game $G_{M}(\varphi)$. Hence: for an IF-formula $\varphi, M \nvdash \models_{\mathrm{t}} \varphi$ does not necessarily imply that $M \| \models_{\mathrm{f}} \varphi$.

A strategy prescribes a choice for a player in every possible position of the game in which it is that player's turn. If we describe every such choice by a function, a strategy consists of a sequence of functions. The arguments of those functions reflect the available input information of the choices. By this correspondence the existence of a winning strategy can be expressed as a $\Sigma_{1}^{1}$-sentence: a statement about the existence of functions satisfying certain first order properties.

In the book [Hintikka 1996], the main focus is on the expressive power of IF-logic by its truth conditions. By the view that the strategic choices of Eloïse correspond to Skolem functions, the formulation of truth conditions as $\Sigma_{1}^{1}$-sentences, is a matter of applying a generalized Skolemization procedure.

First, we discuss some aspects of the focus on truth. Then we will show how falsity conditions can be formulated as $\Sigma_{1}^{1}$-sentences as well.

\section{Focus on truth}

In his book, as in his other work on the subject, Hintikka focuses strongly on when IF-sentences are true. We give three points where this focus is visible. We illustrate these points by a comparison of the simple IF-formula $\forall x \exists y_{/ x}[x=y]$ with the first order formula $\exists y \forall x[x=y]$.

The first point (also pointed out in [Benthem 2000]) is the notion of equivalence: formulas are equivalent if they are true in exactly the same models. In other words: for all IF-sentences $\varphi$ and $\psi$,

$$
\varphi \equiv_{\mathrm{t}} \psi \stackrel{d}{\Leftrightarrow} \text { for all suitable models } M: M \| \models_{\mathrm{t}} \varphi \text { iff } M \| \models_{\mathrm{t}} \psi .
$$

Hintikka explicitly chooses to work with this version of equivalence:

Are two sentences logically equivalent if they are true in the same models [...] or are they to be called so if they are true and false in the same models? In this work, I will consistently opt for the former alternative[...]. ([Hintikka 1996, 65])

We will call this notion of equivalence truth equivalence. Let us define strong equivalence as follows:

$$
\varphi \equiv \psi \stackrel{d}{\Leftrightarrow} \varphi \equiv_{\mathrm{t}} \psi \text { and } \varphi \equiv_{\mathrm{f}} \psi
$$


where $\equiv_{\mathrm{f}}$ is the obvious counterpart of $\equiv_{\mathrm{t}}$ :

$$
\varphi \equiv_{\mathrm{f}} \psi \stackrel{d}{\Leftrightarrow} \text { for all suitable models } M: M \| \models_{\mathrm{f}} \varphi \text { iff } M \| \models_{\mathrm{f}} \psi .
$$

That strong equivalence is indeed stronger than truth equivalence, can already be recognized in simple formulas: the IF-formula $\forall x \exists y_{/ x}[x=y]$ is true in one-element models only, and thereby truth equivalent to the first order sentence $\exists y \forall x[x=y]$. However, the first formula is never false, whereas the second formula is false in all models with at least two elements.

Another issue that makes the focus on truth evident, is related to the issue of equivalence. Every IF-sentence is claimed to have an existential second order-, in other words: $\Sigma_{1}^{1}$-translation. Conversely, every $\Sigma_{1}^{1}$ sentence is said to be translatable into an IF-sentence. (See [Hintikka 1996, 61-63], items (F) and (G).) This back-and-forth translation is crucial for the arguments backing the metalogical properties.

One should be aware, however, of the fact that the translation procedures are only truth-preserving, and disregard the falsity-aspects of the IF-formulas involved. This is demonstrated by the application of the back-and-forth translation to the first order formula $\exists y \forall x[x=y]$ :

\begin{tabular}{|cccc|}
\hline & $\mathrm{IF}$ & & $\Sigma_{1}^{1}$ \\
\hline$(1)$ & $\exists y \forall x[x=y]$ & & \\
$(2)$ & & & $\exists f \forall x[x=f]$ \\
$(3)$ & $\forall x \exists y / x[x=y]$ & & \\
\hline
\end{tabular}

The formulas (1) and (3) share (2) as their truth condition, but where the original formula is false in all models containing at least two elements, the formula that arises after the back-and-forth translation is never false! This example shows in particular that the back-and-forth translation is not closed on the first order fragment of IF-logic, exactly by the fact that falsity conditions are not preserved.

Finally, the focus on truth is also visible in the syntax: Hintikka defines IF-logic from first order formulas in negation normal form, and applies the slash operator only to existential quantifiers and disjunctions. This means that the information restrictions only restrict Eloïse 
in her choice of strategies, and therefore affect only the truth aspect of a formula.

The formula $\exists y \forall x_{/ y}[x=y]$-which is strongly equivalent to our IFexample $\forall x \exists y_{/ x}[x=y]$ : both are true in one element models only, and never false - is for example not an IF-formula in Hintikka's definition of the IF-syntax.

[Hintikka 1996] stresses the importance of the descriptive function of logic: characterization of classes of models by logical formulae.

Given a formula, we can investigate the class of models in which this formula is true by the given semantics. In the case of IF-logic, this comes down to answering the question: given an IF-formula $\varphi$, in which models $M$ does Eloïse have a winning strategy for $G_{M}(\varphi)$ ? But an IFformula not only determines the class of models that make it true, it also determines the class of models that make the formula false. So, one could ask the dual question: given an IF-formula $\varphi$, in which models does Abélard have a winning strategy for $G_{M}(\varphi)$ ?

If $\varphi$ is a first order formula, we know that the class of models in which Abélard has a winning strategy, is exactly the complement of the class of models in which Eloïse has a winning strategy: first order semantical games are determined. For IF-formulas however, the answer to our dual question does not necessarily give us the answer to the first. In any case, as IF-semantical games are in general not determined, the second class is not simply the complement of the first.

We will study how to characterize the class of models in which an IF-formula is false. But first we have to settle some technical issues in order to be able to broaden the perspective of IF-logic from truth-only to truth-and-falsity.

\section{Technicalities}

First, it should be remarked that we will take IF-logic to be closed under (the game theoretical) negation, i.e. if $\varphi$ is an IF-formula, then so is $\sim \varphi$.

In his book, Hintikka is not explicit about this: he defines the syntax from first order formulas already in negation normal form, with the slash applied to existential quantification and disjunction only ([Hintikka $1996,52])$. This would mean that the moves of the player in the role 
of Falsifier would always be of perfect information. Consider for example the negated formula $\sim \forall x \exists y_{/ x}[x=y]$, which can be seen never to be true, and false in one-element models only. Then the weakly equivalent ("truth-equivalent") formula $\exists x \forall y[x \neq y]$ would be an IF-formula (this formula is always false), and not the strongly equivalent formula $\exists x \forall y / x[x \neq y]$.

If one focuses on truth only, imperfect information for the Falsifier is irrelevant. But as we want to include falsity aspects, we choose to allow for the application of the slash to both $\exists$ and $\forall$, and to both $\vee$ and $\wedge$. This enables us to state the following:

Fact 3. 1 (De Morgan's laws for IF-logic). If $\varphi$ is an IF-sentence, write $\varphi[\psi] \equiv \varphi\left[\psi^{\prime}\right]$ to express that if $\psi$ occurs as a subformula of $\varphi$, then the result of replacing $\psi$ with $\psi^{\prime}$ in $\varphi$, is (strongly) equivalent to $\varphi$. We can then state De Morgan's laws for IF-logic as follows:

$$
\begin{aligned}
\varphi\left[\sim\left(\forall x_{/ y} \psi\right)\right] & \equiv \varphi\left[\exists x_{/ y}(\sim \psi)\right] \\
\varphi\left[\sim\left(\exists x_{/ y} \psi\right)\right] & \equiv \varphi[\forall x / y(\sim \psi)] \\
\varphi\left[\sim\left(\psi_{1} \wedge / y \psi_{2}\right)\right] & \equiv \varphi\left[\left(\sim \psi_{1}\right) \vee_{/ y}\left(\sim \psi_{2}\right)\right] \\
\varphi\left[\sim\left(\psi_{1} \vee_{/ y} \psi_{2}\right)\right] & \equiv \varphi\left[\left(\sim \psi_{1}\right) \wedge / y\left(\sim \psi_{2}\right)\right]
\end{aligned}
$$

In fact, these equivalences hold in the strongest sense possible. Not just does there exist a winning strategy for the formula on the left side iff there exists a winning strategy for the one on the right side (for a given player, in a given model). More directly: a winning strategy for the formula on the left side is a winning strategy for the right side and vice versa. This is because the games for the formulas on the left side, are exactly the same games as those for the formulas on the right side.

Informally: the exchange of roles combined with the flipping of the quantifier (or connective) cancels out. This can easily be seen if we code semantical games as games in extensive form. (See for example [Osborne \& Rubinstein 1994] for a general description of games in extensive form, and [Sandu \& Pietarinen 2003], [Sandu \& Pietarinen 2001] or [Dechesne 2005] for the formalisation of semantical games in these terms.)

Corollary 3.1. For every IF-formula $\varphi$ there exists a strongly equivalent IF-formula in negation normal form (NNF), i.e. with negation signs occurring before atomic formulas only.

When formulating the conditions under which a player has a winning strategy, we assume that no information is available on which strategy 
the opponent has chosen to play. In a realistic situation, one could imagine that some strategies of the opponent can be marked as more or less probable (particularly when the game is played repeatedly). But semantical games are abstract games in which no probability, only certainty counts.

We could say that the two players choose their own strategies simultaneously, independently of the other player.

From this follows:

Lemma 3.2. An IF-sentence $\varphi$ in NNF is truth-equivalent with the IF-formula $\varphi^{\prime}$ resulting from $\varphi$ after removing the slashes at universal quantifiers and conjunctions.

E.g.:

$$
\forall x \exists y_{/ x} \forall z / y=\equiv_{\mathrm{t}} \forall x \exists y_{/ x} \forall z \psi .
$$

This lemma enables us to use Hintikka's Skolemization procedure for finding truth conditions. We needed the slashes at universal quantifiers and conjunctions in the process of writing an IF-formula in a (strongly) equivalent negation normal form. But after this process, we can ignore them again when looking for a truth condition. This way, we avoid obtaining an existential second order truth condition with slashed universal quantifiers and connectives (which would not be a $\Sigma_{1}^{1}$-formula because of these slashes).

The dual of the lemma is of course also true, but we will not need it: $\varphi$ is falsity-equivalent to the IF-formula $\varphi^{\prime \prime}$ resulting from $\varphi$ after removing the slashes for existential quantifiers; e.g.

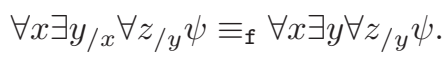

\section{Winning conditions for both players}

[Hintikka 1996, 61] gives an extended Skolemization-procedure to transform an IF-first order sentence $\varphi$ (in NNF) into a $\Sigma_{1}^{1}$-sentence $\Phi$ of the form:

$$
\exists f_{1} \ldots \exists f_{k} \forall x_{1} \ldots \forall x_{l} \xi,
$$

where $\xi$ is a quantifier-free (and slash-free) formula.

By the correspondence between the notion of strategy that emerges from Hintikka's GTS, and the notion of Skolem function, this second 
order sentence $\Phi$ serves as the truth condition for $\varphi$ : for every model $M$ suitable for the language of $\varphi$

$$
M \|=_{\mathrm{t}} \varphi \text { iff } M \models_{\mathrm{sol}} \Phi,
$$

where we use ' $\models$ sol' for satisfaction in semantics for second order logic. Hintikka seems to have intended full semantics for second order logic ([Hintikka 1996, 214, second paragraph]). ${ }^{1}$

We note here that, even though the choice of semantics determines the evaluation of the truth- and falsity conditions, and crucially, the metalogical properties of IF-logic, it does not play a role in the construction of the truth (and falsity) conditions. However, the result we prove in section 6.1 does depend on the choice of full semantics for second order logic.

The following simple observation shows how truth- and falsity conditions are two sides of the same coin:

Lemma 4.1. A falsity condition for an IF-sentence $\varphi$ is a truth condition of $\sim \phi:$

$$
M \| \models_{\mathrm{f}} \varphi \text { iff } M \|=_{\mathrm{t}} \sim \varphi .
$$

This follows easily from the interpretation of negation as role exchange in GTS.

Corollary 4.2. We can express the truth and falsity conditions of an IF-formula $\varphi$ by two $\Sigma_{1}^{1}$-sentences, $\Phi_{\mathrm{t}}$ and $\Phi_{\mathrm{f}}$.

To obtain $\Phi_{\mathrm{f}}$, we first construct an IF-formula that is equivalent to $\sim \varphi$ (by Corollary 3.1), then drop occurring slashes for universal quantifiers (by Lemma 3.2) and finally apply the Skolemization procedure (as described by Hintikka).

As an example, we formulate the truth- and falsity conditions of a formula $\varphi$ that is true exactly in all infinite models (that such formulas exist, is well-known and follows easily from the fact that every $\Sigma_{1}^{1}$-sentence is truth condition of an IF-formula).

Infinity is a typical example of a $\Sigma_{1}^{1}$-definable property of models (as a consequence of the compactness of first order logic, it is not first order definable). The truth condition $\Phi_{\mathrm{t}}$ expresses Dedekind-infinity: the existence of of an injective, non-surjective function on the model of

1. We refer to [Väänänen 2001] for an overview of the consequences of the choice of semantics for the metalogical properties of second order logic. 
the domain. Note that in $\Phi_{\mathrm{t}}, f_{2}$ are $f_{3}$ forced to be interpreted by the same function, by the left-right part of the double arrow, that is injective by the right-left part, and non-surjective by the right conjunct $\left(f_{1}\right.$ is a 0 -ary function symbol, and is used as a Skolem constant). ${ }^{2}$

$$
\begin{aligned}
\varphi & :=\exists z \forall x \forall y \exists u / y \exists v_{/ x}[(x=y \leftrightarrow u=v) \wedge u \neq z] \\
\Phi_{\mathrm{t}} & : \quad \exists f_{1} \exists f_{2} \exists f_{3} \forall x \forall y\left[\left(x=y \leftrightarrow f_{2}(x)=f_{3}(y)\right) \wedge f_{2}(x) \neq f_{1}\right] \\
\sim \varphi & \equiv \forall z \exists x \exists y \forall u / y \forall v_{/ x}[\sim(x=y \leftrightarrow u=v) \vee u=z] \\
\Phi_{\mathrm{f}} & : \quad \exists f_{1} \exists f_{2} \forall z \forall u \forall v\left[\neg\left(f_{1}(z)=f_{2}(z) \leftrightarrow u=v\right) \vee u=z\right]
\end{aligned}
$$

We can now conclude that $\varphi$ is false in $M$ precisely if $M$ is a 1-element model, because only in that case: $M \models \Phi_{\mathrm{f}}$ ).

We warn the reader that any IF-sentence that is true in precisely the class of infinite models, must be undetermined in some finite models. If an IF-sentence would be false in (precisely) all finite models, its falsity condition would be a $\Sigma_{1}^{1}$-sentence expressing finiteness. This is impossible because $\Sigma_{1}^{1}$ is compact.

This shows how we should be careful with the notions like finiteness and infinity (the one is the contradictory negation of the other), in combination with the game theoretical negation and the associated notions of truth and falsity. Because we don't have a contradictory negation in IF-logic, expressibility of infinity does not imply expressibility of finiteness.

\section{$5 \quad$ IF-sentences correspond with $\Sigma_{1}^{1}$-pairs}

With the procedure described above, we have a mechanism to associate with every IF-sentence $\varphi$, a pair $\left(\Phi_{\mathrm{t}}, \Phi_{\mathrm{f}}\right)$ of $\Sigma_{1}^{1}$-sentences, such that for every suitable model $M$ :

$$
M \| \models_{\mathrm{t}} \varphi \Leftrightarrow M \models_{\mathrm{sol}} \Phi_{\mathrm{t}}
$$

and

$$
M \| \models_{\mathrm{f}} \varphi \Leftrightarrow M \models_{\mathrm{sol}} \Phi_{\mathrm{f}} .
$$

The pair $\left(\Phi_{\mathrm{t}}, \Phi_{\mathrm{f}}\right)$ divides the class of all suitable models for the language of $\varphi$ into three disjoint subclasses: the class $\mathcal{M}_{\mathrm{t}}^{\varphi}$ of models satisfying

2. Although the definition of implication for arbitrary IF-formulas is problematic [Hintikka 1996, 138], on the atomic level we can read implication signs as classical material implication. We use the double implication in $\varphi$ just for notational efficiency: ' $(x=y \leftrightarrow u=v)$ ' should be read as ' $(x=y \wedge u=v) \vee(x \neq y \wedge u \neq v)$ '. 
$\Phi_{\mathrm{t}}$, the class $\mathcal{M}_{\mathrm{f}}^{\varphi}$ of models satisfying $\Phi_{\mathrm{f}}$, and finally the class $\mathcal{M}_{\mathrm{u}}^{\varphi}$ of models satisfying neither. We remark that the class $\mathcal{M}_{\mathrm{u}}^{\varphi}$ is not $\Sigma_{1}^{1}$ - but $\Pi_{1}^{1}$-definable. If it is empty, then $\mathcal{M}_{\mathrm{t}}^{\varphi}$ is the complement of $\mathcal{M}_{\mathrm{f}}^{\varphi}$, hence both classes are both $\Sigma_{1}^{1}$ and $\Pi_{1}^{1}$, and thus first order definable. This can only be the case if $\varphi$ is (strongly equivalent to) a first order formula.

For a $\Sigma_{1}^{1}$-sentence $\Phi$, let $\mathcal{M}_{\mathrm{sol}}^{\Phi}$ denote the class of suitable models $M$ in which $\Phi$ is satisfied $\left(M \models_{\text {sol }} \Phi\right)$.

We can now say that, if $\varphi$ is an IF-sentence and $\Phi$ a $\Sigma_{1}^{1}$-sentence, then $\Phi$ is a truth condition for $\varphi$ if $\mathcal{M}_{\mathrm{t}}^{\varphi}=\mathcal{M}_{\mathrm{sol}}^{\Phi}$. Also, $\Phi$ is a falsity condition for $\varphi$ if $\mathcal{M}_{\mathrm{f}}^{\varphi}=\mathcal{M}_{\mathrm{sol}}^{\Phi}$.

On a sentential (propositional) level, we can formulate a nice correspondence between the propositional connectives $\wedge, \vee, \sim$, and the following operations on pairs of $\Sigma_{1}^{1}$-sentences:

\begin{tabular}{|c|c|}
\hline IF-sentence & $\Sigma_{1}^{1} \times \Sigma_{1}^{1}$ \\
\hline$\varphi$ & $\left(\Phi_{\mathrm{t}}, \Phi_{\mathrm{f}}\right)$ \\
$\psi$ & $\left(\Psi_{\mathrm{t}}, \Psi_{\mathrm{f}}\right)$ \\
$\sim \varphi$ & $\left(\Phi_{\mathrm{f}}, \Phi_{\mathrm{t}}\right)$ \\
$\varphi \wedge \psi$ & $\left(\Phi_{\mathrm{t}} \wedge \Psi_{\mathrm{t}}, \Phi_{\mathrm{f}} \vee \Psi_{\mathrm{f}}\right)$ \\
$\varphi \vee \psi$ & $\left(\Phi_{\mathrm{t}} \vee \Psi_{\mathrm{t}}, \Phi_{\mathrm{f}} \wedge \Psi_{\mathrm{f}}\right)$ \\
\hline$\sim \varphi \vee \psi$ & $\left(\Phi_{\mathrm{f}} \vee \Psi_{\mathrm{t}}, \Phi_{\mathrm{t}} \wedge \Psi_{\mathrm{f}}\right)$ \\
\hline
\end{tabular}

The last line of the table gives some insight in the problems we have defining implication for IF-logic. It shows that a definition of implication in terms of game theoretical negation and the disjunction would be stronger than intended. Intuitively, one expects an implication $\varphi \Rightarrow_{\mathcal{G}} \psi$ in GTS to express "if Eloïse has a winning strategy in $G_{M}(\varphi)$, then she has one in $G_{M}(\psi)$ ". This is a (material) implication on the level of strategies, and would correspond to the truth condition: $\neg \Phi_{\mathrm{t}} \vee \Psi_{\mathrm{t}}$. This condition is weaker than the condition $\Phi_{\mathrm{f}} \vee \Psi_{\mathrm{t}}$, because $\mathcal{M}_{\mathrm{f}}^{\varphi} \subseteq\left(\mathcal{M}_{\mathrm{t}}^{\varphi}\right)^{c}$, which for IF-formulas in general is a proper inclusion. More importantly, $\neg \Phi_{\mathrm{t}} \vee \Psi_{\mathrm{t}}$ is in general not $\Sigma_{1}^{1}$, while truth conditions of IF-formulas are always $\Sigma_{1}^{1}$.

As suggested by [Hintikka 1996, 161, item (k)], this problem is solved in so called Extended IF-logic, in which contradictory negation is added as a truth functional operator. Propositional logic with strong and weak negation is one of the topics studied in [Sandu \& Pietarinen 2001].

If we would like to exceed the propositional level, and formulate the truth- and falsity conditions for IF-formulas rather than sentences, the 
treatment of the slashed quantifiers turns out to be a difficult hurdle as of their context dependency. Semantics for IF-formulas with free variables are given in e.g. [Hodges 1997], [Caicedo \& Krynicki 1999].

\section{$6 \quad \Sigma_{1}^{1}$-pairs corresponding with IF-sentences}

In [Caicedo \& Krynicki 1999], the following question is posed: Is it true that for each pair of disjoint $\Sigma_{1}^{1}$-classes $K_{1}, K_{2}$ of structures of the same type there is an IF-sentence $\varphi$ such that $K_{1}=\mathcal{M}_{\mathrm{t}}^{\varphi}$ and $K_{2}=\mathcal{M}_{\mathrm{f}}^{\varphi}$ ? In such case part (b) [of Theorem 5.1 in their paper] would give a quick proof of Craig's interpolation theorem. ([Caicedo \& Krynicki 1999, 30], translated into our notation)

In a recent note of J. Burgess on this question in a logic with Henkin quantifiers ( [Burgess 2003]), the issue is attacked in the reverse direction. Using Interpolation ${ }^{3}$ for $\Sigma_{1}^{1}$, Burgess proves that for any pair of incompatible Henkin sentences $\left(\Phi_{0}, \Phi_{1}\right)$, there is a Henkin sentence $\Theta$ such that $\Theta$ is (truth-)equivalent with $\Phi_{0}$ and $\sim \Theta$ is (truth-)equivalent with $\Phi_{1} \cdot{ }^{4}$ In order to prove this, the class of models is restricted:

To avoid trivialities, in the logic of first-order sentences it is conventional to exclude models with an empty domain, while in the logic of Henkin sentences it will be convenient to exclude models with a one-element domain as well. ([Burgess 2003])

On a closer look, this restriction can be seen to be quite natural for IF-languages: in models with only one element, the slash in IF-formulas is meaningless. We also note that the existence of at least two distinct elements in the models is already implicitly assumed by Hintikka, e.g. in his procedure to eliminate slashed connectives [Hintikka 1996, 52].

Note that under this restriction, the formula $\theta_{0}:=\forall x \exists y_{/ x}[x=y]$ is never (i.e. in no model) true, nor false. We choose not to introduce a new notation for our classes of models to make the restriction, in the rest of this paper, to " $\geq 2$-models" explicit. So: $\mathcal{M}_{\mathrm{t}}^{\theta_{0}}=\mathcal{M}_{\mathrm{f}}^{\theta_{0}}=\emptyset$.

3. More specifically: the fact that for any incompatible pair of $\Sigma_{1}^{1}$-formulas $\Phi, \Psi$ (i.e. $\left.\mathcal{M}_{\text {sol }}^{\Phi} \cap \mathcal{M}_{\text {sol }}^{\Psi}=\emptyset\right)$, there is a first order sentence $\theta$ such that $\mathcal{M}_{\text {sol }}^{\Phi} \subseteq \mathcal{M}_{\text {sol }}^{\theta}$ and $\mathcal{M}_{\text {sol }}^{\Psi} \subseteq\left(\mathcal{M}_{\text {sol }}^{\theta}\right)^{c}$.

4. Here we use the symbol $\sim$ for what Burgess calls contrary negation of a Henkin formula, which is obtained by flipping all quantifiers in the prefix and negating the matrix, thus corresponding to our game theoretical negation. In his note, Burgess uses the symbol $\neg$ for this contrary negation and $\sim$ for contradictory negation. To stay consistent with Hintikka's work, we use the symbols the other way around. 
We can easily translate the result for Henkin sentences into the following theorem about pairs of $\Sigma_{1}^{1}$-sentences and IF-logic:

Theorem 6.1. Let $\Phi$ and $\Psi$ be incompatible $\Sigma_{1}^{1}$-sentences. Then there is an IF-sentence $\theta$ such that $\Phi$ is a truth condition for $\theta$, and $\Psi$ is a falsity condition for $\theta$.

Proof: We use the IF-sentence $\theta_{0}:=\forall x \exists y_{/ x}[x=y]$.

To start the proof, consider the special case where $\mathcal{M}_{\text {sol }}^{\Psi}=\emptyset(\Psi$ is an inconsistent $\Sigma_{1}^{1}$-formula). Applying the translation procedure from $\Sigma_{1}^{1}$ to IF-logic ([Hintikka 1996, 61-63]) to $\Phi$, we find an IF-formula $\varphi$ such that $\mathcal{M}_{\mathrm{t}}^{\varphi}=\mathcal{M}_{\mathrm{sol}}^{\Phi}$. Now take for $\theta:=\varphi \vee \theta_{0}$. This gives us:

$$
\begin{gathered}
\mathcal{M}_{\mathrm{t}}^{\theta}=\mathcal{M}_{\mathrm{t}}^{\varphi \vee \theta_{0}}=\mathcal{M}_{\mathrm{t}}^{\varphi} \cup \mathcal{M}_{\mathrm{t}}^{\theta_{0}}=\mathcal{M}_{\mathrm{t}}^{\varphi} \cup \emptyset=\mathcal{M}_{\mathrm{t}}^{\varphi}=\mathcal{M}_{\mathrm{sol}}^{\Phi} \\
\mathcal{M}_{\mathrm{f}}^{\theta}=\mathcal{M}_{\mathrm{f}}^{\varphi \vee \theta_{0}}=\mathcal{M}_{\mathrm{f}}^{\varphi} \cap \mathcal{M}_{\mathrm{f}}^{\theta_{0}}=\mathcal{M}_{\mathrm{f}}^{\varphi} \cap \emptyset=\emptyset=\mathcal{M}_{\mathrm{sol}}^{\Psi}
\end{gathered}
$$

Now consider the general case. Using the translation procedure of Hintikka, we find IF-sentences $\varphi^{\prime}, \psi^{\prime}$ such that $\mathcal{M}_{\mathrm{t}}^{\varphi^{\prime}}=\mathcal{M}_{\text {sol }}^{\Phi}$ and $\mathcal{M}_{\mathrm{t}}^{\psi^{\prime}}=$ $\mathcal{M}_{\mathrm{sol}}^{\Psi}$. We now form $\varphi:=\varphi^{\prime} \vee \theta_{0}$ and $\psi:=\psi^{\prime} \vee \theta_{0}$, in order to get $\mathcal{M}_{\mathrm{t}}^{\varphi}=\mathcal{M}_{\mathrm{sol}}^{\Phi}, \mathcal{M}_{\mathrm{t}}^{\psi}=\mathcal{M}_{\mathrm{sol}}^{\Psi}$, and $\mathcal{M}_{\mathrm{f}}^{\varphi}=\mathcal{M}_{\mathrm{f}}^{\psi}=\emptyset$.

By Craig's theorem for $\Sigma_{1}^{1}$, because $\Phi$ and $\Psi$ are incompatible, there is a first order sentence $\chi$ such that $\mathcal{M}_{\mathrm{t}}^{\varphi}=\mathcal{M}_{\mathrm{sol}}^{\Phi} \subseteq \mathcal{M}_{\mathrm{t}}^{\chi}$ and $\mathcal{M}_{\mathrm{t}}^{\psi}=$ $\mathcal{M}_{\mathrm{sol}}^{\Psi} \subseteq\left(\mathcal{M}_{\mathrm{t}}^{\chi}\right)^{c}=\mathcal{M}_{\mathrm{f}}^{\chi}(\chi$ is first order $)$.

Now take $\theta:=\varphi \wedge(\sim \psi \vee \chi)$, and we will have the following, to complete the proof:

$$
\begin{gathered}
\mathcal{M}_{\mathrm{t}}^{\theta}=\mathcal{M}_{\mathrm{t}}^{\varphi} \cap\left(\mathcal{M}_{\mathrm{t}}^{\sim \psi} \cup \mathcal{M}_{\mathrm{t}}^{\chi}\right)=\mathcal{M}_{\mathrm{t}}^{\varphi} \cap\left(\mathcal{M}_{\mathrm{f}}^{\psi} \cup \mathcal{M}_{\mathrm{t}}^{\chi}\right)=\mathcal{M}_{\mathrm{t}}^{\varphi} \cap \mathcal{M}_{\mathrm{t}}^{\chi}=\mathcal{M}_{\mathrm{sol}}^{\Phi} \\
\mathcal{M}_{\mathrm{f}}^{\theta}=\mathcal{M}_{\mathrm{f}}^{\varphi} \cup\left(\mathcal{M}_{\mathrm{f}}^{\sim \psi} \cap \mathcal{M}_{\mathrm{f}}^{\chi}\right)=\mathcal{M}_{\mathrm{t}}^{\psi} \cap \mathcal{M}_{\mathrm{f}}^{\chi}=\mathcal{M}_{\mathrm{sol}}^{\Psi}
\end{gathered}
$$

We can conclude that the (strong) expressive power of IF-sentences consists of all pairs of incompatible $\Sigma_{1}^{1}$-sentences.

As Burgess stresses in [Burgess 2003], contrary negation on Henkin sentences (for which we can read game theoretical negation on IF-sentences) "does not correspond to the semantic operation of complementation on classes of models, but further it does not correspond to any semantic operation at all." 
The theorem above shows that there can be many different IF-formulas that are truth equivalent, but pairwise not strongly equivalent. In fact, there are as many of these formulas as there are $\Sigma_{1}^{1}$-definable classes of models disjoint with the (one) class of models in which they are all true.

To illustrate this, for every $n \in \mathbb{N}$, let $\psi_{n}$ be a first order formula expressing that the domain contains at least $n$ elements (for example, we can take $\left.\psi_{3}=\exists x_{1} \exists x_{2} \exists x_{3}\left[x_{1} \neq x_{2} \wedge x_{2} \neq x_{3} \wedge x_{3} \neq x_{1}\right]\right)$, and let $\varphi$ be an IF-formula that is true in infinite models only (e.g. the formula $\varphi$ on page 309 of this paper). Define $\varphi_{n}:=\varphi \wedge \psi_{n}$. Then for every $n$, $\mathcal{M}_{\mathrm{t}}^{\varphi_{n}}=\mathcal{M}_{\mathrm{t}}^{\varphi}$ (i.e. the class of all infinite models), while for all $m$ with $n<m: \mathcal{M}_{\mathrm{f}}^{\varphi_{n}} \varsubsetneqq \mathcal{M}_{\mathrm{f}}^{\varphi_{m}}$ (i.e. the classes of models with less than $n$ and $m$ elements respectively).

\section{Reflections on strategies and Skolemization}

The particular outcome of a run of a game $G_{M}(\varphi)$ is not what is relevant for GTS. We are not even interested in particular winning strategies; we are only interested to know whether one of the players has a winning strategy, and if so, which player.

We could say that, by formulating truth- and falsity conditions as above, we have given a 'strategic' form of semantical games: Eloïse can force a win in $G_{M}(\varphi)$ if she can give an interpretation for the Skolem functions such that $M$ extended with these interpretations satisfies the first order part of the $\Sigma_{1}^{1}$-sentence $\Phi_{\mathrm{t}}$; and similarly for Abélard and $\Phi_{\mathrm{f}}$.

A game of perfect information in which the moves do not consist of giving interpretations for variables, but of giving interpretations for Skolem functions (only by Eloïse) is proposed in [Väänänen 2002].

Underlying the construction of our truth and falsity conditions, is the view that Skolemization is the natural formalization of strategies in semantical games. We did not go into the details of this procedure, but there is one aspect we would like to discuss here: the order in which the Skolemization steps are applied.

Skolemization consists of a step-by-step replacement of existentially quantified variables by terms of the form $f\left(x_{1}, \ldots, x_{n}\right)$, where $f$ is a new function symbol, and $x_{1}, \ldots, x_{n}$ the variables in the scope of whose quantification the existential quantification occurs; see e.g. [Fitting 1996, 
section 7.11$]^{5}$.

For first order logic, Skolemiziation is usually applied 'outside-in', meaning that we start by replacing the outermost existential quantification. As a result, the arguments of the Skolem-functions are only universally quantified variables. For example, $\forall x \exists z \exists y[R(x, y, z)]$ becomes $(\exists g)$ $\forall x \exists y[R(x, y, g(x))]$, which then becomes $(\exists f \exists g) \forall x[R(x, f(x), g(x))]$. If we would have applied the procedure 'inside-out', we would have got: $\forall x \exists z \exists y[R(x, y, z)]$ becomes $(\exists f) \forall x \exists z[R(x, f(x, z), z)]$, which then becomes $(\exists g \exists f) \forall x[R(x, f(x, g(x)), g(x))]$. The latter formula is of course equivalent to $(\exists g \exists h) \forall x[R(x, h(x), g(x))]$, hence in this case, the order of the Skolemization procedure does not make a difference. In general: for first order formulas, the order of the Skolemization doesn't matter.

However, the IF-formula $\forall x \exists z \exists y_{/ x}[x=y]$ (proposed in [Hodges 1997, example 5.2]) shows us that in IF-logic the order of the (generalized) Skolemization procedure is relevant. As result of the outside-in procedure it gives $\exists f \exists g \forall x[x=f]$ (where $f$ is a Skolem constant), while the inside-out procedure first gives $\exists f \forall x \exists z[x=f(z))]$, and then $\exists g \exists f \forall x[x=$ $f(g(x))]$. Game theoretically, Skolemizing outside-in allows only previous moves of the opponent as arguments of the strategy functions, while Skolemizing inside-out allows all previous moves.

Hintikka confirms his choice for the (classically more usual) outsidein procedure, by assuming the provision "that moves connected with existential quantifiers are always independent of earlier moves with existential quantifiers" ([Hintikka 1996, 63]). This results in the gametheoretically counterintuitive assumption that each player has forgotten his/her own previous moves. ${ }^{6}$

In [Hodges 1997] and [Caicedo \& Krynicki 1999], we find semantics for (more general) IF-languages, without this provision. The strategy functions in these semantics can be seen to correspond with inside-out Skolemization, as they use all previously quantified variables as arguments for the functions.

On formulas such as Hodges's formula above, these semantics therefore differ from Hintikka's semantics with the provision. Also, semantics

5. For (slashed) disjunctions in IF-formulas, a similar replacement is part of Hintikka's generalized Skolemization procedure. We do not discuss this part of the procedure separately, also because it implies that disjunctions may be replaced by existential quantifications.

6. Or, alternatively, that we should see each player rather as a team, where each member of the team knows some previous moves of the other team, but none of the moves of his own team. 
corresponding to inside-out Skolemization give us the means to characterize infinity of the domain of a model in a more compact way: the formula

$$
\exists u \forall x \exists y \exists z / x[x=z \wedge y \neq u]
$$

then gets the truth condition (with $k$ a Skolem constant):

$$
\exists k \exists g \exists f \forall x[x=f(k, g(k, x)) \wedge g(k, x) \neq k]
$$

expressing that there is a function $g_{k}=g(k, \ldots)$ that is injective (because it has a left-inverse $f_{k}=f(k, \ldots)$ ), and non-surjective (because $k$ is outside the range of $g_{k}$ ). This example ([Caicedo \& Krynicki 1999, example 1.4]) contains less quantifiers and connectives then the formula $\varphi$ given on page 309 of this paper.

Dropping the provision (or equivalently, switching from outside-in to inside-out Skolemization) still delivers $\Sigma_{1}^{1}$-truth conditions. The expressive power of IF-logic as a whole therefore does not depend on the order chosen (see also [Caicedo \& Krynicki 1999, theorems 4.2 and 4.3]).

\section{Reflections on game theoretical negation}

In this paper we have focused on falsity aspects of IF-formulas, a topic closely related to the subject of game theoretical negation (as witnessed by lemma 4.1).

The definition of game theoretical negation as role exchange, makes the formula $\varphi \vee \sim \varphi$ a statement expressing the determinacy of the games $G_{M}(\varphi)$. We know that games for IF-sentences $\varphi$ are in general not determined, so $\varphi \vee \sim \varphi$, the 'law' of the excluded middle, is not a logical law for IF-logic.

This reminds us of course of negation in intuitionistic logic, which is also too strong to make the law of the excluded middle hold. We would like to point out that this eye-catching shared property does not automatically make IF-logic and intuitionistic logic related.

First, as easily as we see a shared property of the negations in the two logics, we can also find a singificant difference. In intuitionistic logic, the law of the excluded middle can be seen to be equivalent to the cancellation of double negation $(\neg \neg A \rightarrow A)$, and conversely. In IFlogic however, $\sim \sim \varphi$ is clearly equivalent to $\varphi$, by the fact that a double role exchange ("turning the game board around twice in a row") has no significant effect to the semantical game. 
Another important difference is that at the atomic level, game theoretical negation is equal to classical negation: the winner of the game is determined by a purely classical evaluation of the atomic formula in the given model. This gives every run of a game a winner. In intuitionistic logic, it is possible that we do not have a proof nor a refutation for an atomic proposition: atomic formulas may be undetermined.

Furthermore, game theoretical negation can be seen to coincide with classical negation not only at the atomic level, but on all unslashed formulas: i.e. for all classical first order sentences $\varphi$ :

$$
\begin{gathered}
M \models \varphi \stackrel{A C}{\rightleftarrows} M \| \models_{\mathrm{t}} \varphi, \text { and: } \\
M \models \neg \varphi \stackrel{A C}{\rightleftarrows} M \| \models_{\mathrm{t}} \sim \varphi
\end{gathered}
$$

This follows from the axiom of choice, because this makes first order formulas (interpreted classically) equivalent to their Skolemizations, i.e. the truth conditions of these formulas in GTS. In the game theoretical definition of truth, the axiom of choice is incorporated ([Hintikka 1996, 40]).

We see game theoretical negation and its behavior in GTS for IFlogic, as an extension of classical negation rather than related to intuitionistic negation. The truth value gap introduced by the extension of the game theoretic negation beyond first order logic is in some sense unavoidable: truth and falsity conditions are incompatible and both $\Sigma_{1}^{1}$, while $\Sigma_{1}^{1}$ is not closed under classical negation (in other words: the collection of $\Sigma_{1}^{1}$-definable classes of models is not closed under complements). However, if we would like to regard game theoretical negation as an extension of the operation that classical negation defines on the first order definable classes of models to the $\Sigma_{1}^{1}$-definable classes, we have to realize that the game theoretical negation does not correspond to any operation at al, as shown by the result of Burgess (Theorem 6.1).

\section{Conclusions}

We showed how a falsity condition for an IF-formula $\varphi$, can be obtained as a truth condition for $\sim \varphi$. We characterize an IF-formula not only by its truth (as is usually the case in Hintikka's work), but by both truth and falsity, hence by a pair of $\Sigma_{1}^{1}$-sentences. A result of Burgess shows that this gives us a correspondence between IF-sentences and incompatible pairs of $\Sigma_{1}^{1}$-sentences. 
The procedures to obtain truth and falsity conditions, rely on a Skolemization procedure. We explained that the order of the Skolemization steps does make a difference in IF-logic, unlike in first order logic: it reflects whether or not a player is assumed to forget his/her own previous moves. This difference does however not alter the expressive power of IF-logic as a whole.

The result of Burgess demonstrates the curious fact that game theoretical negation does not correspond with any operation on classes of models. Furthermore, we argued that game theoretical negation should not be associated with intuitionistic negation to easily, just because both make the 'law' of the excluded middle fail. IF-logic with GTS incorporates some unconstructive elements that make the game theoretical negation more classical than intuitionistic.

For future research, it would be interesting to study the effect of interpreting truth and falsity conditions constructively rather than classically. This would not only mean that we restrict the existential second order quantifications to range, for example, over recursive functions, as suggested in [Hintikka 1996, chapter 10], but we would also have to interpret the connectives and predicates in a constructive way.

\section{References}

BENTHEM, JOHAN VAN

2000 Hintikka Self-Applied: an essay on the epistemic logic of imperfect information games, preliminary version of [Benthem 2005].

2005 The epistemic logic behind IF-games, in R.E. Auxier and L.E. Hahn, (eds), The Philosophy of Jaakko Hintikka, volume 30 of $L i$ brary of Living Philosophers, Southern Illinois University. Forthcoming.

Burgess, John

2003 A remark on Henkin sentences and their contraries, Notre Dame Journal of Formal Logic, 44(3), 185-188.

Caicedo, Xavier and Krynicki, Michal

1999 Quantifiers for reasoning with imperfect information and $\Sigma_{1}^{1}$ logic, in Contemporary Mathematics, volume 235, American Mathematical Society, 1999: 17-31.

\section{Dechesne, Francien}

2005 Games, Set, Maths: formal investigations into logic with im- 
perfect information, Ph.D. thesis, SOBU: Tilburg University and Technische Universiteit Eindhoven, 2005.

\section{Fitting, MeLvin}

1996 First order logic and automated theorem proving, Graduate texts in computer science. Berlin: Springer Verlag, second edition, 1996.

HiNTIKKA, JAAKKO

1996 The Principles of Mathematics Revisited, Cambridge University Press, 1996.

\section{HodGes, WiLfRID}

1997 Compositional semantics for a language of imperfect information, Bulletin of the IGPL, 5 (4), 539-563, 1997.

Osborne, Martin J. \& Rubinstein, Ariel

1994 A Course in Game Theory, MIT Press, 1994.

Sandu, Gabriel and Pietarinen, Ahti

2001 Partiality and games: Propositional logic, Logic Journal of the IGPL, 9(1), 101-121, 2001.

SAndu, Gabriel and Pietarinen, Ahti

2003 Informationally independent connectives, in G. Mints, and R. Muskens, (eds), Logic, Language and Computation, volume 9, 2341. Stanford: CSLI publications, 2003.

VÄÄNÄNEN, JOUKO

2001 Second order logic and the Foundations of Mathematics Bulletin of Symbolic Logic, 7, 504-520, 2001.

2002 On the semantics of informational independence, Logic Journal of the IGPL, 10(3), 339-352, 2002. 